\title{
A utilização do conhecimento científico na área da Nutrição para a tecnologia de informação e comunicação (TIC) PODCAST
}

The use of scientific knowledge in the area of Nutrition for PODCAST information and communication technology (ICT)

El uso del conocimiento científico en el área de Nutrición para la tecnología de información y comunicación (TIC) PODCAST

\section{Resumo}

Objetivo: Apresentar a utilização da TIC podcast como potencial ferramenta, baseada em prévia investigação científica, para impulsionar o desenvolvimento de profissionais nutricionistas. Metodologia: Foi realizado estudo transversal, quantitativo e descritivo, com profissionais nutricionistas do território brasileiro, de ambos os sexos. A coleta de dados foi online a partir da aplicação de questionário desenvolvido no "Formulários Google" e auto preenchido pelo voluntário. Foram coletados dados relacionados ao perfil sociodemográfico e ocupacional, tipo predominante de rede social consumida, condições de saúde e aspectos relacionados à área de atuação profissional. Resultados: Dos 115 voluntários, 95\% são mulheres, 48,7\% informaram receber mais que 3 salários mínimos, 46,95\% recebem de 1 até 3 salários mínimos, além de dados coletados sobre questões que enfatizavam sobre a jornada de trabalho, satisfação com a profissão, área de atuação, desafios e motivações para a mudança de profissão. A partir da análise dessas informações foi realizada a escolha dos 10 primeiros temas para a criação dos podcasts, estreia e apresentação, sociedade do cansaço e produtividade, desvalorização profissional, ser mulher e seus diversos papeis/funções, estigma do peso e pressão estética na profissão, estresse ocupacional e síndrome de burnout, compulsão alimentar, desenvolvimento de carreira, área de nutrição e os influenciadores digitais, e impunidade. Conclusão: Conclui-se que após a investigação científica, os dados demonstraram uma insatisfação dos nutricionistas com a profissão, desejando maior valorização profissional e que o podcast apresenta um potencial de ser uma ferramenta verossímil, capaz de atender às necessidades laborais.

Palavras-chave: Nutricionistas; Mídias sociais; Comunicação e divulgação científica; Desenvolvimento humano.

\footnotetext{
Abstract

Goal: Introduce the usage of the TIC podcast as a potential tool, based on prior scientific research, to boost the development of professional nutritionists. Methodology: a transversal, quantitative and descriptive study was carried out with professional nutritionists from the Brazilian territory, of both sexes. Data was collected online through a
} 
questionnaire developed in "Google Forms" and self-filled by the volunteer. Data related to sociodemographic and occupational profile, predominant type of social network consumed, health conditions and aspects related to the area of professional activity were collected. Results: Out of the 115 volunteers, $95 \%$ are women, $48.7 \%$ reported receiving more than 3 minimum wages, $46.95 \%$ receive from 1 to 3 minimum wages, in addition to data collected on issues that emphasized the labor journey, satisfaction with the profession, occupation area, challenges and motivations for changing the profession. Based on the analysis of this information, the first 10 themes were chosen for the creation of podcasts, debut and presentation, society of tiredness and productivity, professional devaluation, being a woman and its various roles/functions, weight stigma and aesthetic pressure in the profession, occupational stress and burnout syndrome, binge eating, career development, nutrition and digital influencers, and impunity. Conclusion: It is concluded that after scientific investigation, the data showed a dissatisfaction of nutritionists with the profession, desiring greater professional appreciation and that the podcast has the potential to be a credible tool, capable of meeting labor needs.

Keywords: Nutritionists; Social networks; Scientific communication and diffusion; Human development.

\section{Resumen}

Objetivo: Presentar el uso del TIC podcast como una herramienta potencial, basada en investigaciones científicas previas, para impulsar el desarrollo de los nutricionistas profesionales. Metodología: Se realizó un estudio transversal, cuantitativo y descriptivo con nutricionistas profesionales del territorio brasileño, de ambos sexos. La recolección de datos se llevó a cabo online a partir de la aplicación de un cuestionario desarrollado en "Google Forms" y respondido por el voluntario. Se recogieron datos relacionados con el perfil sociodemográfico y ocupacional, tipo predominante de red social consumida, condiciones de salud y aspectos relacionados con el área de actividad profesional. Resultados: De los 115 voluntarios, el 95\% son mujeres, el 48,7\% reportó recibir más de 3 salarios mínimos, el 46,95\% recibe de 1 a 3 salarios mínimos, además de datos recolectados sobre temas que enfatizan la jornada laboral, satisfacción con la profesión, área de especialización, desafíos y motivaciones para el cambio de profesión. Con base en el análisis de esta información, se eligieron los primeros 10 temas para la creación de podcasts, son ellos: debut y presentación, sociedad del cansancio y la productividad, devaluación profesional, ser mujer y sus diversos roles/funciones, estigma sobre peso y presión estética en la profesión, estrés laboral y síndrome de burnout, atracones de comida, desarrollo profesional, nutrición e influencers digitales, e impunidad. Conclusión: Se concluye que luego dela investigación científica, los datos evidenciaron un descontento de los nutricionistas con la profesión, deseando una mayor valoración profesional y que el podcast tiene el potencial de ser una herramienta creíble, capaz de satisfacer las necesidades laborales.

Palabras clave: Nutricionistas; Redes sociales; Comunicácion y difusión científicas; Desarrollo humano.

\section{Introdução}

A Nutrição é uma ciência recente e que se encontra em constante evolução. A profissão de nutricionista somente foi reconhecida e regulamentada em 1967, por meio da Lei $n^{\circ} .5276$ / 1967, posteriormente revogada pela Lei $\mathrm{n}^{\circ} 8234$ / 1991 (Brasil, 1967; 1991) e desde então houveram grandes mudanças e evoluções no conhecimento e na forma como os nutricionistas interagem entre si e com seu público.

Os desafios profissionais vivenciado pelos nutricionistas amplia o objetivo e a complexidade do seu trabalho, ficando clara a urgência de estabelecer interfaces na nutrição com outras áreas do conhecimento. Uma das principais queixas é a desvalorização profissional, insatisfação salarial, ausência de capacitação e desenvolvimento adequados e acessíveis, bem como a ausência de um plano de desenvolvimento de carreira e salários, além de outros desafios diários enfrentados por esses profissionais.

Neste sentido, pode-se dizer que a área da saúde envolve situações que exigem a prática integrada de saberes, habilidades técnicas, atitudes e capacidade de tomada de decisões. A formação de profissionais voltada apenas para o desempenho técnico específico da sua área de atuação, não pode mais ser aceita pelas instituições de ensino superior, neste final de século. Sua preocupação deve voltar-se para a formação do profissional cidadão, competente técnica e cientificamente, mas sobretudo com uma ampla visão da realidade em que vai atuar e com elementos para transformá-la. Com a preocupação de transmitir conhecimentos específicos sobre a profissão, os cursos superiores na área da saúde não têm conseguido formar profissionais cidadãos com uma prática determinada por valores éticos, humanos e com capacidade para pensar a construção de uma nova sociedade (Amorim, et al., 2001). 
No contexto da formação em saúde, o uso das tecnologias de informação e comunicação (TICs) tem se destacado como uma ferramenta pedagógica capaz de democratizar e expandir as oportunidades educacionais e propiciar uma educação aberta e continuada em saúde (Curioni, et al., 2013).

As redes sociais podem ser consideradas como as ferramentas de comunicação mais importantes do século XXI. Uma das razões para essa popularidade se dá pelo seu potencial no cenário educacional e por oferecer à população meios para interagir e expressar conhecimento e informações entre si, sendo útil para a aprendizagem e a criação de conhecimento. Além disso, as redes sociais já são apontadas como facilitadoras de aprendizagem e multiplicação de ensino (Borges, 2020).

As TICs correspondem a todas as tecnologias que facilitam a coleta, o processamento, o armazenamento e a troca de informações por meio do uso da comunicação eletrônica. Além disso, podem ser entendidas como um conjunto de recursos tecnológicos integrados entre si, que proporcionam a automação e comunicação dos processos de negócios, serviços públicos, pesquisa, os científicos e os de ensino aprendizagem, através das funções de hardware, software e telecomunicações (Curioni, et al., 2013; ABDI, 2010).

No estudo de Curioni, et al. (2013) que revisou as experiências do uso de TICs na área da Nutrição e a reflexão sobre seus impactos e desafios, verificou um contínuo avanço e difusão do uso das TICs na área de nutrição, as quais são utilizadas também como ferramentas de educação em saúde. Esses avanços permitem amplo acesso à educação em nutrição para profissionais que, de outra forma, teriam dificuldade de participar presencialmente de cursos e capacitações.

Entre as novas tecnologias de aprendizagem está inserido o podcast, um arquivo de áudio que pode ser combinado com vídeo para a abordagem de temáticas variadas. Criado em 2004, o podcast é uma tecnologia de oralidade que possui caráter educativo e estimula atividades lúdicas (Freire, 2017).

Visto como um espaço de comunicação, o podcast estabelece um diálogo democrático, contribuindo na construção da perspectiva de mundo do sujeito por meio do constante ensino-aprendizado de modo crítico e reflexivo, pois o podcast atende as diferentes formas de aprendizagens, já que proporciona um acesso rápido e fácil ao conhecimento com o intuito de promover informações de cuidado, autocuidado, ensino e aprendizagem, desenvolvimento pessoal e profissional, uma vez que a informação alcança a comunidade com uma metodologia que ultrapassa a informação textual. Cada arquivo de áudio do podcast é chamado de episódio e devem possuir um tempo relativamente pequeno para se obter maior adesão dos ouvintes (Costa, et al., 2021).

Essa tecnologia educacional se caracteriza por ser aberta e pela liberdade produtiva, fato que potencializa a ampliação do conteúdo e a possibilidade de debate de temas diversos e relevantes (Freire, 2017). Assim, representa uma importante ferramenta na redução da assimetria comunicacional, sendo porta-voz de questões cotidianas ou relacionadas a grupos minoritários com incipiente visibilidade nos grandes meios de comunicação (Costa, et al., 2021).

Vale ressaltar que os podcasts são feitos, muitas vezes, por percepções sociais e midiática, sem a base de prévios trabalhos científicos. A escolha dos temas norteadores dos episódios dos podcasts, na maioria dos casos, ocorre em função do jornalismo ou até mesmo por profissionais de saúde, mas não tem a base de um artigo científico que direcione os temas, que é justamente a proposta deste trabalho.

Destaca-se que é importante sim que os podcasts tenham uma percepção social, mas eles não podem perder de perspectiva que é extremamente necessário um prévio trabalho científico para detectar os problemas daquele público a quem se deseja atingir, para melhor contribuição social.

Dentre as problemáticas levantadas pela pesquisa, destacam-se os desafios da capacitação, o trabalho de desenvolvimento e empoderamento de nutricionistas, mostrando que esses profissionais podem ser conscientes de suas escolhas e seus destinos, considerando suas distintas necessidades, faz-se iminente contribuir no enfrentamento dos desafios do ser profissional nutricionista e no modo de conduzi-los. 
Por relevância da pesquisa entende-se abrangência do tema e o impacto (aplicabilidade do resultado) no contexto científico, inovação, clínico, social, tecnológico, educacional ou econômico (Estrela, 2018).

Constitui-se como objetivo geral deste trabalho, apresentar a utilização da TIC podcast como potencial ferramenta, baseada em prévia investigação científica, para impulsionar o desenvolvimento de profissionais nutricionistas.

\section{Metodologia}

Foi realizado estudo transversal, quantitativo e descritivo, com profissionais nutricionistas do território brasileiro, de ambos os sexos. A abordagem quantitativa apresenta dados coletados de uma determinada amostra para levantamento, comparação e análise e interpretação dos dados coletados, contribui de forma ímpar para abordagem analítica dos dados e tabulação dos resultados obtidos no pro cesso metodológico (Moresi, et al., 2003).

A coleta de dados ocorreu, no período de janeiro a abril de 2021 (período de 90 dias), por meio da aplicação de questionário desenvolvido pela ferramenta "Formulários Google" e auto preenchido pelo voluntário. O recrutamento foi realizado pelo compartilhamento do link do questionário feito na plataforma Google forms e o mesmo foi enviado via e-mail, pelas redes sociais "WhatsApp", "facebook" e "Instagram", em grupos e canais específicos de profissionais nutricionistas como também aos Conselhos Regionais de Nutricionistas (CRN) para compartilhamento e divulgação em seus canais de comunicação e divulgação. Aqueles que aceitaram participar do estudo, de forma voluntária e anônima, assinaram o termo de consentimento livre e esclarecido (TCLE), para o prosseguimento na pesquisa. Foram coletados dados no intuito de obter informações para conhecer a população do estudo, como dados relacionados ao perfil sociodemográfico e ocupacional, tipo predominante de rede social consumida, condições de saúde, aspectos relacionados a área de atuação profissional, como satisfação com o trabalho, desenvolvimento, qualidade de vida e bem estar como forma para melhor compreensão dos anseios e dificuldades vivenciadas por esses profissionais.

Foram estabelecidos como critérios de inclusão: nutricionistas de ambos os gêneros, estar devidamente registrado no CRN da sua região, não estar gestante e que aceitaram assinar o TCLE.

Os dados coletados na pesquisa foram base norteadora para a escolha dos temas dos podcasts. Os temas escolhidos para o planejamento dos dez primeiros episódios foram: apresentação do projeto, objetivos e motivações, sociedade do cansaço e produtividade, desvalorização profissional, ser mulher e seus diversos papéis e funções, estigma do peso e pressão estética na profissão, estresse ocupacional e síndrome de burnout, compulsão alimentar, desenvolvimento de carreira, nutrição e os influenciadores digitais e impunidade no ambiente de trabalho.

Após 10 episódios, baseado na audiência, será feito novo questionário para ver: primeiro, potencialidades e fragilidades dos programas que foram expostos; segundo, futuros temas a serem abordados ou rebordados.

$\mathrm{O}$ presente trabalho respeitou todas as orientações no que diz respeito às pesquisas com seres humanos, com aprovação no Comitê de Ética em Pesquisa (CEP) da Universidade Federal do Rio de Janeiro - Campus Macaé (CAAE: 39723920.8.0000.5699).

\section{Resultados e Discussão}

Os dados foram analisados por meio de estatística descritiva, de modo que foi possível fazer a contabilização e a descrição dos dados encontrados.

Para compilação dos dados coletados, utilizou-se a análise temática de conteúdo, a qual estabelece um conjunto de técnicas de análise da comunicação buscando identificar o núcleo de sentido produzido na mensagem. Para a realização dessa 
análise são necessárias algumas etapas, tais como: leitura seletiva do material, organização das respostas dos participantes, seleção das respostas, identificação das unidades de contexto, estabelecimento das categorias de análise (FARIA, 2017)

A pesquisa contou com a participação de 118 voluntários, sendo 3 excluídos do estudo, por não atenderem aos critérios de inclusão, contabilizando 115 participantes.

Na Tabela 1, pode-se observar o perfil sociodemográfico dos nutricionistas entrevistados.

Tabela 1: Perfil sociodemográfico dos pesquisados.

\begin{tabular}{|c|c|}
\hline Variáveis & $\mathrm{n}(\%)$ \\
\hline \multicolumn{2}{|l|}{ Gênero } \\
\hline Masculino & $3(2,61)$ \\
\hline Feminino & $112(97,39)$ \\
\hline \multicolumn{2}{|l|}{ Regiões do Brasil } \\
\hline Norte & $3(2,61)$ \\
\hline Centro-Oeste & $20(17,4)$ \\
\hline Nordeste & $14(12,17)$ \\
\hline Sudeste & $66(57,39)$ \\
\hline Sul & $12(10,43)$ \\
\hline \multicolumn{2}{|l|}{ Idade } \\
\hline 21 a 30 anos & $42(36,52)$ \\
\hline 31 a 40 anos & $52(45,22)$ \\
\hline 41 a 50 anos & $18(15,65)$ \\
\hline$>50$ anos & $3(2,61)$ \\
\hline \multicolumn{2}{|l|}{$\begin{array}{l}\text { Estado Civil } \\
\end{array}$} \\
\hline Solteiro & $49(42,61)$ \\
\hline Casado & $57(49,56)$ \\
\hline Divorciado & $18(15,65)$ \\
\hline Viúvo & $3(2,61)$ \\
\hline \multicolumn{2}{|l|}{ Filhos } \\
\hline Sim & $54(49,96)$ \\
\hline Não & $61(53,04)$ \\
\hline \multicolumn{2}{|l|}{ Cor/Raça } \\
\hline Amarelo (a) & $7(6,09)$ \\
\hline Branco (a) & $69(60)$ \\
\hline Indígena & 0 \\
\hline Preto/Pardo/Negro (a) & $37(32,17)$ \\
\hline Prefiro não responder & $2(1,74)$ \\
\hline \multicolumn{2}{|l|}{ Renda Mensal } \\
\hline Até 3 salários mínimos & $54(46,95)$ \\
\hline <3 salários mínimos & $56(48,7)$ \\
\hline Prefiro não responder & $5(4,35)$ \\
\hline
\end{tabular}

Fonte: Dados da pesquisa (2021).

Com relação ao perfil dos nutricionistas, mais de 95\% são do sexo feminino, com idade média de 34,15 \pm 7,31 anos, predominante da raça branca (60\%). De acordo com o Conselho Federal de Nutricionistas (CFN, 2016), 94,1\% dos nutricionistas brasileiros são do sexo feminino. A predominância da mulher no exercício da profissão de nutricionista também foi descrita nos estudos de Araújo (2018) e Rocha et al. (2017), onde a amostra foi exclusivamente feminina (100\%). Em relação à variável sexo, os resultados corroboram o fato de ser uma profissão predominantemente feminina, característica inerente às origens históricas desse profissional no Brasil, sendo divulgado como um curso apropriado a moças e que continua se reproduzindo nos dias atuais (Andrade, 2012; Lima, 2020). 
Entretanto, apesar de ser uma profissão majoritariamente feminina, a inserção do gênero masculino tem se intensificado na profissão com o passar dos anos. O CFN (2016) também mostrou que ao relacionar com a área de atuação, os profissionais do sexo feminino referem atuar predominantemente nas áreas de Alimentação Coletiva e Nutrição Clínica.

Com relação à renda mensal dos nutricionistas, 48,7\% informaram receber mais que 3 salários mínimos, 46,95\% recebem de 1 até 3 salários mínimos e 4,35\% preferiram não responder à pergunta. Segundo a classificação de classe social, e de acordo com o Instituto Brasileiro de Geografia e Estatística (IBGE, 2020), a maioria dos nutricionistas pertence à classe econômica $\mathrm{C}$ e $\mathrm{D}$, onde os rendimentos são entre quatro e dez salários mínimos e entre dois e quatro salários mínimos, respectivamente. Inclusive, a insatisfação salarial foi algo muito citado pelos participantes do estudo quanto à pergunta sobre os motivos para a mudança de profissão. A insatisfação salarial é algo discutido há anos no universo da Nutrição, que apesar das conquistas obtidas ao longo dos anos, ainda luta por melhores condições salariais para a classe.

De acordo com a Federação Nacional de Nutricionistas (FNN), em 2021, o piso salarial nacional estabelecido foi de R \$ 3.067,12 para uma jornada de 44 horas semanais. Para os nutricionistas que desempenham a função de Responsável técnico (RT), na área de Alimentação Coletiva, tem o acréscimo de 10\% em cima do valor do piso salarial vigente. Pesquisa realizada pelo CFN (2016) com nutricionistas do Brasil, identificou que dos 1083 entrevistados, 3,05\% (n=33) recebem até 1 salário mínio, 32,5\% (n=352) recebem até 3 salários mínimos e 64,45\% (n=698) ganham mais que 3 salários m. Os que recebem maior renda, provavelmente, são os que atuam a mais tempo na profissão, possuem maior tempo de empresa ou podem estar atuando em cargos de gestão, a nível de coordenação, gerência ou direção operacional, ou mesmo na área da docência, além de possuírem um plano de carreira bem desenvolvido.

Há anos o CFN articula projetos de lei sobre o piso salarial e jornada de trabalho de nutricionistas, onde no ano de 2021 o ponto já se encontra em tramitação na Câmara dos Deputados, sendo protocolado o Projeto de Lei n 1578/2021, que pode alterar a Lei $\mathrm{n}^{\circ} 8.234 / 91$, que regulamenta a profissão do nutricionista. O texto trata do piso salarial (no valor de cinco salários mínimos) e da jornada de 30 horas semanais, com remuneração proporcional caso a jornada exceda as 30 horas, não podendo ultrapassar as 44 horas semanais.

O questionário que foi aplicado aos profissionais nutricionistas também continha perguntas para avaliar a percepção do próprio nutricionista sobre sua profissão, composto por questões que enfatizavam sobre a jornada de trabalho, satisfação com a profissão e área de atuação e motivações para a mudança de profissão. Um resultado que chamou a atenção foi a insatisfação de muitos com a profissão e a desvalorização salarial. A Tabela 2 mostra os questionamentos dos participantes para a mudança da profissão

Tabela 2: Motivos relatados pelos nutricionistas para mudança de profissão.

\begin{tabular}{|c|}
\hline $\begin{array}{l}\text { Principais motivos para mudança de profissão/área } \\
\text { de atuação }\end{array}$ \\
\hline $\begin{array}{c}\text { Remuneração } \\
\text { Oportunidade / Gostar de outra área }\end{array}$ \\
\hline $\begin{array}{l}\text { Ambiente de trabalho: Estresse / Falta de } \\
\text { reconhecimento e incentivo / Plano de carreira / } \\
\text { Excesso de cobrança / Jornada de trabalho / Condições } \\
\text { de trabalho / Desgaste físico e emocional }\end{array}$ \\
\hline $\begin{array}{l}\text { Remuneração e ambiente de trabalho } \\
\text { Qualidade de vida e bem estar }\end{array}$ \\
\hline
\end{tabular}


Oliveira e Medeiros (2019) ressaltam que para ter uma boa qualidade de vida no trabalho é necessário existir motivação. O colaborador satisfeito é aquele que percebe a sua importância dentro da organização. O gestor tem o papel de ter uma visão diferenciada e identificar qualidades em cada funcionário, dando-lhes a oportunidade de demonstrarem o seu valor e com isso contribuir melhor com a empresa, a partir disso o colaborador vai se sentir mais seguro e ter um bom desempenho que é o fator principal da estabilidade no trabalho.

Almeida et al. (2015) ainda ressalta que devem ser implantadas condutas que se iniciem no processo de formação ao ingressar na empresa, com foco na autoeficácia e no desenvolvimento de habilidades de enfrentamento, podendo incluir programas de prevenção abordando as causas e consequências da insatisfação com o ambiente de trabalho.

Referente à formação profissional, Sartor e Alves (2019) afirmam que nutricionistas que atuam, especialmente na área de refeições coletivas pouco buscam formação acadêmica complementar e a dedicação se baseia ao exercício diário da profissão no mercado de trabalho. Para Rocha et al. (2017), uma capacitação maior do nutricionista iria promover uma melhoria de resultados e melhoria na execução das tarefas em geral, entre elas as da área gerencial como também as questões relacionadas a valorização salarial.

Pela análise dos dados obtidos com o presente estudo, demonstra que a profissão do Nutricionista é uma área que anseia um programa de desenvolvimento e fortalecimento. Isso pode ser feito se apropriando e utilizando as ferramentas que estão prejudicando a ciência da Nutrição, como as mídias sociais, para o benefício da mesma. Ou seja, se apropriar das TICs, de forma congruente, com integridade e ética como um caminho para a comunicação e divulgação científica, com a finalidade de desenvolvimento desses profissionais. Entretanto, é fundamental que o discurso esteja alinhado com a ação sincronizada. A realização de ações com esse objetivo podem ser uma forma de influenciar profissionais a aprimorar seus conhecimentos e desenvolver competências visando crescimento e novas oportunidades.

É necessário se pensar em criar estratégias de capacitação e desenvolvimento profissionais alinhando o conhecimento científico a educação profissional. O que se vê é um novo cenário para a produção e apropriação do conhecimento que começa a surgir, particularmente ao fim do século XX. Uma necessidade que se faz presente é uma mudança na forma de se fazer e comunicar ciência, conectando-a à tecnologia, mercado e sociedade, o que levaria a um novo modo de organização do conhecimento (Borges, 2020).

Hoje em dia, quando grande parte da população brasileira se informa por redes sociais, especialmente pelo WhatsApp (Agência Brasil, 2019). Os próprios entrevistados informaram usar o WhatsApp (47,30\%), como principal rede social consumida, seguida do uso do Instagram (45,95\%).

A incorporação das TICs pode apoiar tais processos, atendendo aos interesses da sociedade atual. O uso de tais tecnologias vem se consolidando no setor educacional, com a promessa de cenários inovadores, apoiados pela potencialidade dos espaços virtuais. A web torna-se, gradativamente, espaço comum de intercâmbio de informações, democratizando o acesso às informações, assim, como sua universalização (Silva, 2010), além de acarretar a minimização dos efeitos deletérios da anticiência.

Também é importante constatar que a pandemia Covid-19 acelerou o movimento de disseminação do uso das tecnologias da Quarta Revolução Tecnológica, intensificando a disseminação de tecnologias digitais remotas na área da saúde e da vida social em geral (Magalhães \& Couldry, 2020).

Diante da crise e dos desafios atuais, impõem-se profundas transformações estruturais que abram espaço para as forças expansivas que estão gravemente refreadas, em detrimento das vastas possibilidades de melhora humana oferecidas pelos avanços científicos e tecnológicos (Gadelha, 2021).

Uma das TICs que vem ganhando adesão no meio educacional é o uso de podcast, entendido como um canal com grande potencial de influência, que faz parte cada vez mais dos hábitos de informação atuais. Como mídia, é um instrumento 
de disseminação de informações e veículos para aproximar a ciência da sociedade. Como uma mídia em crescimento, seu potencial pode ser de grande valia para os esforços de divulgação científica (Curioni et al., 2021).

É importante compreender que o podcast tem um grande potencial de divulgar a publicação de seu conteúdo, extrapolando o universo sonoro; já que hospedado em ambiente digital, pode vir acompanhado de imagens, textos, links, resumos, críticas, comentários anteriores e posteriores ao consumo do produto, o que fazem parte do entorno em que se convive com podcasting (Primo, 2006).

Também é importante saber que o desenvolvimento de um produto requer compreender onde ele será lançado e os fatores internos e externos que podem interferir em seu desempenho. Assim, foi utilizada a análise SWOT, sigla em inglês que se refere à strengths, weakness, opportunities e threats: uma matriz que analisa forças e fraquezas no ambiente interno do produtor e oportunidades e ameaças no macroambiente para a avaliação do uso da TIC podcast (Dutra, 2014).

Na Figura 1, pode observar a matriz SWOT criada para análise do uso do podcast como ferramenta educacional para o desenvolvimento de nutricionistas.

Figura 1: Matriz SWOT para uso da TIC PODCAST.

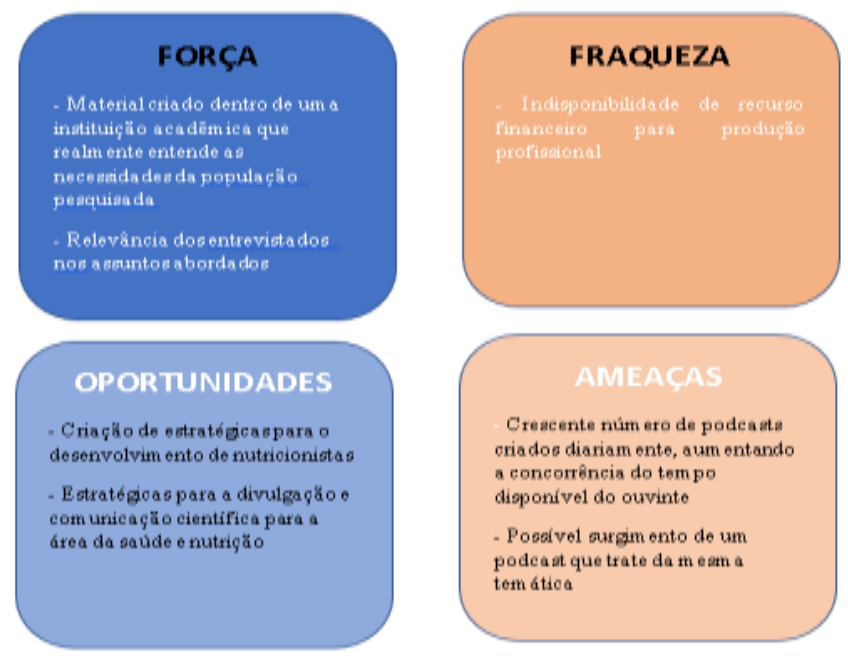

Fonte: Autores (2021).

A aplicação do modelo SWOT permite fazer cruzamentos entre oportunidades e forças e ameaças e fraquezas para analisar o cenário como um todo e os riscos ao lançar um determinado produto. Nesse sentido, é possível observar uma grande viabilidade pela ausência de similares encontrados e pela relevância do conteúdo a ser criado.

O crescimento do podcast como meio de informação disponibiliza aos usuários uma cartela de temática variada. Seja individual ou coletivo, anônimo ou canais de comunicação já estabelecidos, observa-se a criação de novos nichos e segmentos que permite ao usuário diversas possibilidades de conteúdo. Com uso consolidado, vem se apresentando como ferramenta de educação viável, além de ser desenvolvido de maneira simples, possibilitando sua criação sem grandes investimentos de recursos financeiros e com grande poder de alcance.

Para Primo (2006), essa horizontalidade na forma de comunicar e interagir no ambiente digital interconectado é algo que se assemelha ao "boca a boca" na interação presencial, quando participantes de uma rede se comunicam em outra, espalhando informação e multiplicando conteúdo. $\mathrm{O}$ autor ainda afirma que essa interconexão de subsistemas pode produzir grandes efeitos sociais, sem que haja controle ou orquestração do Estado ou da grande mídia. 
Vale destacar que os profissionais da comunicação enfatizam que o público concede maior audiência a formatos modernos e adequados a seu cotidiano, e que a produção de conteúdo precisa considerar, não obstante, classe, região, nível de instrução e qualquer característica de diferentes nichos (Malta, 2017).

Desta forma, ao se pensar em intervir e evoluir na vida coletiva, é que se assevera ser fundamental realizar a divulgação de uma maneira mais ampla e efetiva da pesquisa científica.

Com base nos resultados da pesquisa científica, os temas a serem desenvolvidos nos podcasts estão esquematizados no Quadro 1.

Quadro 1: Estrutura temática e justificativa para os primeiros episódios.

\begin{tabular}{|c|c|c|}
\hline EPISÓDIOS & TEMA & $\begin{array}{l}\text { JUSTIFICATIVA PARA } \\
\text { O TEMA }\end{array}$ \\
\hline $1^{\circ}$ EPISÓDIO & $\begin{array}{l}\text { ESTREIA E APRESENTAÇÃO DO } \\
\text { PROJETO }\end{array}$ & $\begin{array}{c}\text { Resultados da investigação } \\
\text { científica com os } \\
\text { nutricionistas } \\
\end{array}$ \\
\hline $2^{\circ}$ EPISÓDIO & $\begin{array}{l}\text { SOCIEDADE DO CANSAÇO E } \\
\text { PRODUTIVIDADE }\end{array}$ & $\begin{array}{c}\text { Estresse ocupacional atinge } \\
73,04 \% \text { dos entrevistados } \\
68,70 \% \text { alegaram trabalhar } \\
\text { mais de } 8 \text { horas/dia }\end{array}$ \\
\hline $3^{\circ}$ EPISÓDIO & $\begin{array}{l}\text { DESVALORIZAÇÃO } \\
\text { PROFISSIONAL }\end{array}$ & $\begin{array}{l}\text { 48,7\% alegaram receber }< \\
\text { 3salários mínimos } \\
\text { Uma das perguntas com alta } \\
\text { pontuação ( } 405 \text { pontos): } \\
\text { "As poucas perspectivas de } \\
\text { crescimento na carreira têm } \\
\text { me deixado angustiado" }\end{array}$ \\
\hline $4^{\circ}$ EPISÓDIO & $\begin{array}{c}\text { SER MULHER E SEUS } \\
\text { DIVERSOS PAPÉIS/FUNÇÕES }\end{array}$ & $\begin{array}{l}\text { 97,39\% dos participantes } \\
\text { eram do sexo feminino; } \\
\text { 49,96\% tem filhos } \\
\text { Uma das motivações para } \\
\text { mudança de profissão e área } \\
\text { de atuação }\end{array}$ \\
\hline $5^{\circ}$ EPISÓDIO & $\begin{array}{l}\text { ESTIGMA DO PESO E PRESSÃO } \\
\text { ESTÉTICA NA PROFISSÃOO }\end{array}$ & $\begin{array}{c}\text { Dados do IMC: } \\
28,69 \% \text { Sobrepeso e } \\
26,09 \% \text { Obesidade } \\
\end{array}$ \\
\hline $6^{\circ}$ EPISÓDIO & $\begin{array}{l}\text { ESTRESSE OCUPACIONAL E } \\
\text { SÍNDROME DE BURNOUT }\end{array}$ & $\begin{array}{c}\text { Nível de estresse } \\
\text { ocupacional apresentado } \\
\text { pelos avaliados: } 30,43 \% \\
\text { nível de estresse alto e } \\
42,61 \% \text { nível de estresse } \\
\text { moderado } \\
\end{array}$ \\
\hline $7^{\circ}$ EPISÓDIO & COMPULSÃO ALIMENTAR & $\begin{array}{l}56,52 \% \text { alegaram descontar } \\
\text { suas frustações na comida }\end{array}$ \\
\hline $8^{\circ}$ EPISÓDIO & $\begin{array}{l}\text { DESENVOLVIMENTO DE } \\
\text { CARREIRA }\end{array}$ & $\begin{array}{l}\text { Pergunta que obtive maior } \\
\text { pontuação ( } 412 \text { pontos): } \\
\text { "Tenho me sentido } \\
\text { incomodado com a } \\
\text { deficiência nos treinamentos } \\
\text { para a capacitação } \\
\text { profissional." }\end{array}$ \\
\hline $9^{\circ}$ EPISÓDIO & IMPUNIDADE & $\begin{array}{c}\text { Resultados da investigação } \\
\text { científica }\end{array}$ \\
\hline 10 EPISÓDIO & $\begin{array}{l}\text { ÁREA DA NUTRIÇÃO E OS } \\
\text { INFLUENCIADORES DIGITAIS }\end{array}$ & $\begin{array}{c}\text { Proposta do conjunto do } \\
\text { trabalho } \\
\text { Conclusão da primeira } \\
\text { temporada }\end{array}$ \\
\hline
\end{tabular}

Fonte: Autores (2021).

Para o desenvolvimento dos episódios dos podcasts serão convidados, pessoas com experiência profissional na área de pelo menos 5 anos, bem como ser atuante nas mídias sociais para o debate e explanação nas áreas temáticas escolhidas, dentre 
elas: ambiente de trabalho, psicologia organizacional, saúde, síndrome de burnout, estresse ocupacional, desenvolvimento de carreira, compulsão alimentar e mídias sociais.

\section{Conclusão}

Após a investigação científica, os dados demonstraram uma insatisfação dos nutricionistas com a profissão, desejando maior valorização profissional. Com isso, elaborou-se a ferramenta do podcast contendo os principais temas: sociedade do cansaço e produtividade, desvalorização profissional, ser mulher e os diferentes papéis desenvolvidos, estigma do tempo e pressão estética relacionada à profissão, estresse ocupacional e síndrome de burnout, compulsão alimentar, desenvolvimento de carreira, impunidade e a área da nutrição e os influenciadores digitais.

Conclui-se que o podcast apresenta um potencial de ser uma ferramenta verossímil, capaz de atender às necessidades laborais.

Estudos que unam o uso do conhecimento científico às tecnologias da informação e comunicação são cada vez mais necessários para a inserção destas ferramentas no processo de desenvolvimento, contribuindo para um aperfeiçoamento dos profissionais da área da nutrição. Sugere-se a realização de mais estudos referente à temática, possibilitando o acompanhamento e avaliações periódicas do desenvolvimento destes profissionais, e da eficácia do uso destas novas ferramentas tecnológicas e educacionais.

\section{Agradecimentos}

Agradecimento à toda equipe da pesquisa.

\section{Referências}

AGÊNCIA BRASIL. (2019). Pesquisa aponta que 79\% dos brasileiros se informam pelo whatsapp. https://agenciabrasil.ebc.com.br/

Almeida, D. M., Ibdaiwi, T. K. R., Lopes, L. F. D., Costa, V. M. F., \& Possamai, L. O. (2015). Estresse ocupacional na perspectiva dos bombeiros da cidade de Santa Maria/RS. Revista de Carreiras e Pessoas (ReCaPe). 5(1).

Amorim, S. T. S. P. D., Moreira, H., \& Carraro, T. E. (2001). A formação de pediatras e nutricionistas: a dimensão humana. Revista de Nutrição, 14 , 111-118

Andrade, R. M. (2012). Qualidade de vida no trabalho dos colaboradores da empresa Farben S/A indústria química. Trabalho de Conclusão de Curso (TCC) em Nutrição. Universidade do Extremo Sul Catarinense. Santa Catarina.

Araújo, L. R. G. D. (2018). Avaliação da qualidade de vida no trabalho e nível de estresse dos nutricionistas gestores de unidades de alimentação coletiva. Trabalho de Conclusão de Curso (TCC) em Nutrição. Atlas.

Agência Brasileira de Desenvolvimento Industrial (ABDI). (2010). Cadernos Temáticos - Tecnologias de Informação e Comunicação -TIC, Sistemas Aplicados a Saúde Humana. ABDI. 209p.

Brasil, Lei n ${ }^{\circ}$ 8.234. Regulamenta a profissão de Nutricionista e determina outras providências. 17 de setembro de 1991.

Brasil, Lei $\mathrm{n}^{\circ}$ 5.276. Regulamenta a profissão de Nutricionista e determina outras providências. 24 de abril de 1967.

Borges, D. O. (2020). "E aí, pesquisador, comunicar pra quê?": o podcast como estratégia e mídia para a divulgação da ciência. Dissertação de mestrado em Tecnologias, Comunicação e Educação. Universidade Federal de Uberlândia

Conselho Federal De Nutricionistas (CFN). (2016). Perfil das (os) nutricionistas no Brasil. http://pesquisa.cfn.org.br/

Conselho Federal De Nutricionistas (CFN). (2021). Perfil das (os) nutricionistas no Brasil. Dados do primeiro trimestre de 2021. http://pesquisa.cfn.org.br/

Costa, S. D. S. P., de Souza Andrade, C. G., \& Oliveira, C. V. (2021). Desafios De Ser Mulher: Uso Do Podcast Como Meio De Divulgação Científica. Atas de Ciências da Saúde. 11(1), 19.

Curioni, C. C., Brito, F. D. S. B., \& Boccolini, C. S. (2013). O uso de tecnologias de informação e comunicação na área da nutrição. Jornal Brasileiro de TeleSSaúde, 2(3), 51-59.

Dutra, D. V. (2014). A análise SWOT no Brand DNA Process: um estudo da ferramenta para aplicação em trabalhos em Branding. Dissertação (mestrado). Universidade Federal de Santa Catarina. Florianópolis 
Research, Society and Development, v. 10, n. 13, e597101321563, 2021

(CC BY 4.0) | ISSN 2525-3409 | DOI: http://dx.doi.org/10.33448/rsd-v10i13.21563

Estrela, C. (2018). Metodologia científica: Ciência, ensino, pesquisa. Artes Médicas.

Faria, B. A. S. D. (2017). Fatores que influenciam na valorização do nutricionista atuante na área da alimentação coletiva em Cuiabá, MT. Trabalho de Conclusão de Curso (TCC) em Nutrição. Universidade Federal de Mato Grosso. Cuiabá.

Federal Nacional de Nutricionistas (FNN). (2021). Tabela de Honorários Nutricionistas 2021. https://www.fnn.org.br/.

Freire, E. P. A. (2017). Podcast: breve história de uma nova tecnologia educacional. Educação em Revista, 18(2), 55-71.

Gadelha, C. G. (2021). O Complexo Econômico-Industrial da Saúde 4.0: por uma visão integrada do desenvolvimento econômico, social e ambiental. Cadernos do Desenvolvimento, 25.

Instituto Brasileiro de Geografia e Estatística. (2020). Instituto Brasileiro de Geografia e Estatística.

Lima, G. K. M. D., Gomes, L. M. X., \& Barbosa, T. L. D. A. (2020). Qualidade de Vida no Trabalho e nível de estresse dos profissionais da atenção primária. Saúde em Debate, 44, 774-789.

Magalhães, J. C., \& Couldry, N. (2020). Tech Giants Are Using This Crisis to Colonize the Welfare System. Jacobin, 27 abr.

Malta, D. C., Bernal, R. T. I., Lima, M. G., Araújo, S. S. C. D., Silva, M. M. A. D., Freitas, M. I. D. F., \& Barros, M. B. D. A. (2017). Doenças crônicas não transmissíveis e a utilização de serviços de saúde: análise da Pesquisa Nacional de Saúde no Brasil. Revista de Saúde Pública, 51.

Moresi, E. (2003). Metodologia da pesquisa. Brasília: Universidade Católica de Brasília,108 (24), 5

Oliveira, G. S., \& de Medeiros Sousa, H. (2019). Qualidade de vida no trabalho: fatores que influenciam a produtividade nas organizações. Revista Campo do Saber, 4(2).

Primo, Alex. (2006). O aspecto relacional das interações na Web 2.0. In: XIXX Congresso Brasileiro de Ciências da Comunicação: Brasília. Anais

Rocha, G. G., Miyagi, A. M. C., Guimarães, L. I. G., Cardoso, V. D. L., Matias, A. C. G., Spinelli, M. G. N., \& Abreu, E. S. (2017). Caracterização de Nutricionistas de unidades de alimentação e nutrição terceirizadas: dados demográficos, perfil de atuação e percepção de bem estar. Rev Simbio-Logias, 9(12), 53-64.

Sartor, J., \& Alves, M. K. (2019). Percepção do perfil de liderança do nutricionista gestor em unidades de alimentação e nutrição. Saúde e Desenvolvimento Humano, 7(3), 13-19.

Silva, T. O. T. (2010). A Efetividade da Educação a distância na formação de profissionais de saúde: uma análise a partir da inserção no mercado de trabalho. [Effectiveness of e-Learning in the qualificaton of healthcare professionals: analysis from insertion into the work market.]. Essay (Master's Degree). Centro Universitário UMA 